\title{
3-D controlled source electromagnetic modelling based on unstructured mesh using finite element method
}

\author{
Yalu Wang \\ Institute of Geology and Geophysics, CAS \\ Beijing, China \\ wangyalu310@163.com
}

\author{
Qingyun Di \\ Institute of Geology and Geophysics, CAS \\ Beijing, China \\ qydi@mail.iggcas.ac.cn
}

\begin{abstract}
The forward modeling and inversion study on Controlled-Source Audio-Frequency Magnetotelluric method is very important to field work and interpretation of field data. One dimension forward modeling and inversion study has gotten lots of interest over the years and the technology has now been well developed. However, a wrong result may be obtained when the model varies laterally by using $1 \mathrm{D}$ numerical modeling, given that both the source used by CSAMT and the actual model are of $3 D$ character. Hence, there is an urgent need to develop threedimension (3D) forward modeling and inversion technique. To simulate complex geological model, this paper does study on three-dimension finite element modelling based on unstructured grid. We solved the primary field induced by the background model (1D) and the secondary field induced by the anomalous body, respectively, so as to avoid the singularity of the source and reduce the computation area. The algorithm was validated to modeling layered model by comparing its result with the result obtained from integral equation (IE) method. Then, numerical modeling was performed for a single conductive model and the electromagnetic response was analyzed.
\end{abstract}

Keywords-CSAMT; three dimension; finite element; unstructured grid

\section{INTRODUCTION}

CSAMT method has been widely used since it was developed in the 1970s. There have been lots of researches on the data processing and explanation of CSAMT method since then. Although the forward modeling and inversion technique used for one-dimensional study is well developed, however, the source used for this technique is of limited length and the actual geo-electric structure being mapped are usually not one-dimensional body. Hence, wrong result may be obtained by carrying out $1 \mathrm{D}$ numerical modeling. Therefore, there is an urgent need for the development of 3D forward modeling and inversion technique.

The modeling of 3D electromagnetic field started overseas in the 1970s. Hohmann ${ }^{[1]}$ applied the integrated method to 3D MT modeling in 1975 for the first time, and many other researchers have since studied the application of IE method in EM modeling. Unsworth, Wannamaker, Mackie etcetera did lots of researches on the finite element (FE) method and the finite difference (FD) method ${ }^{[2-4]}$. Among these modeling techniques, only the FE method requires the use of flexible gridding system which makes it an effective method in dealing with complex 3D model. Some of the achievements on 3D modeling of electromagnetic field using the FE method have also been published by domestic researchers. Wang Ruo etc carried out preliminary study on 3D modelling using the Galerkin method ${ }^{[5]}$. The accuracy of the solution obtained from the study was improved on by adding the divergence condition forcedly. Xu Zhifeng ${ }^{[6]}$ etc overcame the numerical instability in 3D electromagnetic finite element simulation by adding a penalty term. In further study, Zhang Jifeng ${ }^{[7]}$ etc used the electric field vector technique to derive solution for the 3D boundary value problem.

However, the above mentioned studies on 3D FE method were developed based on structured grids, which may cause large discretization error along the boundary and may result in wrongful interpretation of the subsurface target. Hence, the unstructured gridding system can be used to eliminate the discretization error. Several researches on the applying this technique to three-dimension DC resistivity modeling and MT modeling have been published recently ${ }^{[8-9]}$.

Therefore, in this paper, we developed 3D CSAMT forward modeling algorithm based on the unstructured mesh. The boundary problem for EM secondary field was derived at first. Then, the unstructured gridding system was introduced. The algorithm was validated by modeling two canonical models and comparing the result obtained with that of the IE method.

\section{THE BOUNDARY PROBLEM}

The electromagnetic field excited by horizontal electric dipole source was computed in this paper. Assuming that the time factor is $e^{-i \omega t}$, the equation array which controls the electromagnetic field can be displayed in the diffusion format, where the displacement current is ignored.

$$
\begin{aligned}
& \nabla \times \vec{E}=i \omega \mu_{0} \vec{H} \\
& \nabla \times \vec{H}=\sigma \vec{E}+\vec{J}_{s}
\end{aligned}
$$

$\vec{E}$ is the electric field, $\vec{H}$ is the magnetic field. $\mu_{0}$ is permeability, $\omega$ is angular frequency, $\sigma$ is the conductivity, $\vec{J}_{s}$ is the current density of the source. (2):

We can obtain the double curl equation of $\vec{E}$ from (1) and 


$$
\nabla \times \nabla \times \vec{E}=i \omega \mu_{0}\left(\sigma \vec{E}+\vec{J}_{s}\right)
$$

The electric field and magnetic field in any medium should obey (3). Assuming that $\vec{E}_{p}$ is the background electric field of $1 D$ model, $\vec{E}$ is the total electric field of 3D model and $\vec{E}_{s}$ is the pure secondary electric field of the 3D anomalous body embedded in the 3D model. Hence, the equation for total electric field $\vec{E}$ is represented by (3) and the background electric field $\vec{E}_{p}$ should obey the equation written as follow:

$$
\nabla \times \nabla \times \vec{E}_{p}=i \omega \mu_{0}\left(\sigma_{0} \vec{E}+\vec{J}_{s}\right)
$$

The secondary electric field $\vec{E}_{s}$ can be obtained by finding the difference between $\vec{E}$ and $\vec{E}_{p}$. So the formula which $\vec{E}_{s}$ should obey can be obtained by differentiating (4) from (3):

$$
\nabla \times \nabla \times \vec{E}_{s}=i \omega \mu_{0} \sigma \vec{E}_{s}+i \omega \mu_{0} \Delta \sigma \vec{E}_{p}
$$

$\Delta \sigma$ is the difference between the conductivity of the anomalous body and the background model. Because the computational range is much larger than the anomalous body and the secondary field is weak, hence, the assumption that the secondary field is almost equal to zero is reasonable.

Then the boundary problem which the secondary electric field obey is displayed as follow, and $\Gamma$ represent the boundary of the computation boundary.

$$
\left\{\begin{array}{l}
\nabla \times \nabla \times \vec{E}_{s}=i \omega \mu_{0} \sigma \vec{E}_{s}+i \omega \mu_{0} \Delta \sigma \vec{E}_{p} \\
\vec{E}_{s}=0 \quad \text { on } \Gamma
\end{array}\right.
$$

After adding the divergence condition into the equation, the boundary problem is rewritten as follow:

$$
\left\{\begin{array}{l}
\frac{\partial^{2} E_{s x}}{\partial y^{2}}+\frac{\partial^{2} E_{s x}}{\partial z^{2}}-\frac{\partial^{2} E_{s y}}{\partial y \partial x}-\frac{\partial^{2} E_{s z}}{\partial z \partial x}+i \omega \mu \sigma E_{s x}=i \omega \mu \Delta \sigma E_{p x} \\
\frac{\partial^{2} E_{s y}}{\partial x^{2}}+\frac{\partial^{2} E_{s y}}{\partial z^{2}}-\frac{\partial^{2} E_{s x}}{\partial x \partial y}-\frac{\partial^{2} E_{s z}}{\partial z \partial y}+i \omega \mu \sigma E_{s y}=i \omega \mu \Delta \sigma E_{p y} \\
\frac{\partial^{2} E_{s z}}{\partial x^{2}}+\frac{\partial^{2} E_{s z}}{\partial y^{2}}-\frac{\partial^{2} E_{s x}}{\partial x \partial z}-\frac{\partial^{2} E_{s y}}{\partial y \partial z}+i \omega \mu \sigma E_{s z}=i \omega \mu \Delta \sigma E_{p z} \\
E_{s x}=E_{s y}=E_{s z}=0 \quad \text { on } \Gamma
\end{array}\right.
$$

\section{UNSTRUCTURED MESH}

Gridding is the first step of EM forward modeling by FE method. The advantage of modeling by FE method is that it's capable of modeling complex geology models, but the common structured gridding method is not competent enough. On the other hand, the unstructured gridding technique is flexible, making it competent for the modeling of complex geological models. Lots of algorithms have been used to build unstructured mesh automatically. However, in this paper, we used the Tetgen software developed by Hang Si to grid the models used in this paper.

\section{VALIDATION}

The code was validated by modeling two canonical models and comparing the obtained result with that of the IE method.

Fig. 1 displays a three-layer model. The origin of coordinates locates in the middle of computation range. The model ranges from $-5000 \mathrm{~m}$ to $5000 \mathrm{~m}$ along both the $\mathrm{x}$ direction and $\mathrm{y}$ direction. Along the $\mathrm{z}$ direction, the thickness of the air layer is $5000 \mathrm{~m}$, while the conductivity is $10^{-6} \mathrm{~s} / \mathrm{m}$. The first layer is thick of $300 \mathrm{~m}$ and the conductivity is $0.01 \mathrm{~s} / \mathrm{m}$. the second layer is thick of $300 \mathrm{~m}$, while the conductivity is $0.1 \mathrm{~s} / \mathrm{m}$. The thickness of the 3th layer is $4400 \mathrm{~m}$, and the conductivity is $0.01 \mathrm{~s} / \mathrm{m}$. The source locates along $\mathrm{y}=-3000 \mathrm{~m}$, and its length is $1000 \mathrm{~m}$, the current is $10 \mathrm{~A}$. There are 16 frequencies ranging from $2^{-2} \mathrm{~Hz}$ to $2^{13} \mathrm{~Hz}$.

Fig. 2 shows the apparent resistivity and impedance phase comparison curves between the FE method and the IE method. The red dot represents the result of FE method while the blue curve represents the result obtained from using the IE method. The curves show that the results were consistent with those obtained from using the IE method. The obtained error margin was less than $3 \%$. This suggests that our algorithm can be used to compute the response of three dimension models.

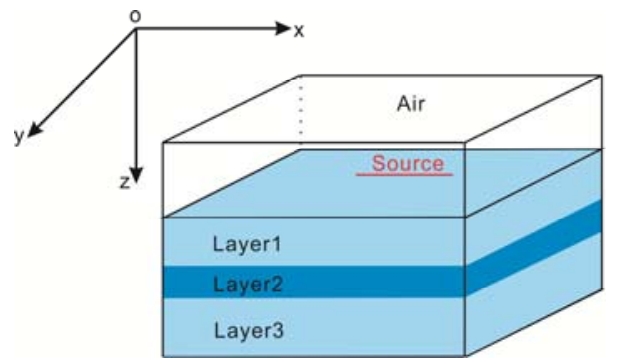

Fig. 1. The three-layer model
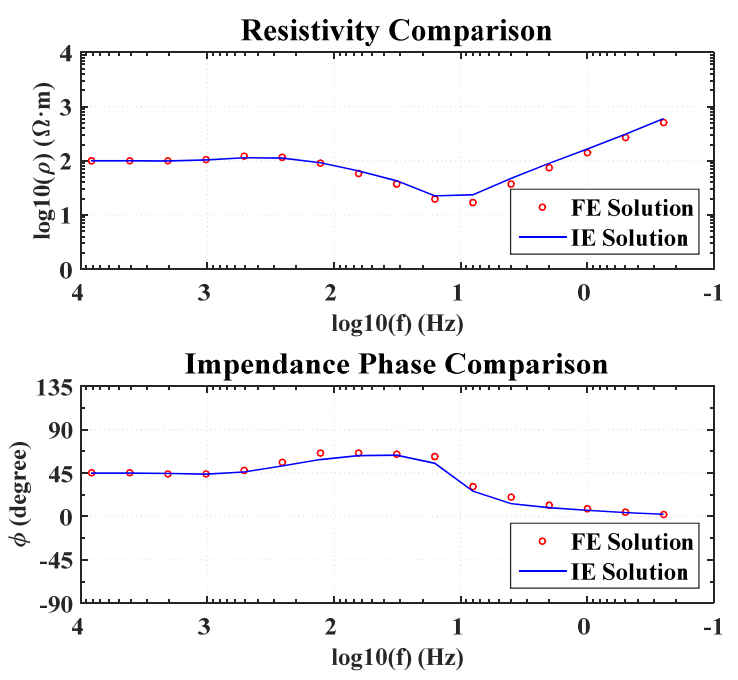

Fig. 2. the resistivity and impedance phase comparison curve 


\section{EXAMPLE}

Fig. 3 shows a single conductive anomaly model. The origin of the coordinate system was the center of the anomalous body projected to the surface. The background model was $5000 \mathrm{~m}$ thick, while its conductivity was $0.01 \mathrm{~s} / \mathrm{m}$. The cubic anomalous body was $300 \mathrm{~m}$ (Length)* $300 \mathrm{~m}$ (Width)* 300m (Height). The depth of the anomalous body was $300 \mathrm{~m}$ and the conductivity was $0.1 \mathrm{~s} / \mathrm{m}$. The computation area was the same as the three-layer model.

The source was located along $y=-3000 \mathrm{~m}$, the length of which was $1000 \mathrm{~m}$, and the current was $1 \mathrm{~A}$. The survey area was located above the anomalous body. 9 lines were set along the y coordinate between the distances of $-800 \mathrm{~m}$ to $800 \mathrm{~m}$, using a traverse-traverse separation of $200 \mathrm{~m}$. 60 points were sited on each of the lines, which had a uniform length of $1800 \mathrm{~m}$, ranging from $-900 \mathrm{~m}$ to $900 \mathrm{~m}$.

Fig. 4 shows the apparent resistivity pseudo-section of the profile $y=0 \mathrm{~m}$. The horizontal axis of the pseudo-section represents the $x$ coordinate of the survey area, while the vertical axis is the apparent depth. The apparent depth was obtained from the concept of skin depth. The red dashed box represents the shape of the anomalous body. It's obvious that the response obtained by the finite element method reflects the shape and location of the anomalous body, especially the vertical location and extent of the body.

Fig. 5 shows the apparent resistivity curve of the profile $\mathrm{y}=0 \mathrm{~m}$. This reflects the presence of the conductive body, especially interm of its horizontal position and lateral extent.

To analyze the responses of the body on different survey lines and the effect of the distance between the source and the survey area on the responses, five horizontal slice images were obtained from the 3D volume. The y coordinates of the slice figures shown in Fig. 6 are -400 m, $-200 m, 0 m, 200 m, 400 m$ respectively, while the $y=0$ profile is the main profile and the other 4 lines are sidelines. The horizontal axis of the slices represents the $\mathrm{x}$ coordinate of the survey area, while the vertical axis represents the apparent depth.

Fig. 6 shows that the main profile reflects the value, location and extent of the anomalous body more better than other profiles. On the lines which are closer to the source, the repsonse are not that obvious. And the nearer the lines are located to the source, the repsonses the smaller. This is because the medium under these lines is the half-space. On the lines which are farer away from the source, the repsonses are obvious. The extent of the parturbation on these lines were larger than that of the main profile, and the center point of the conductive area is more deeper. This is because of the shadow effect of the electromagnetism ${ }^{[10]}$.

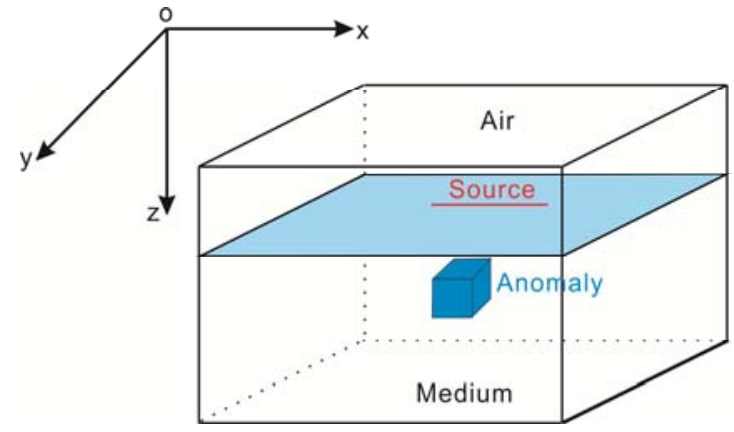

Fig. 3. the single conductive model

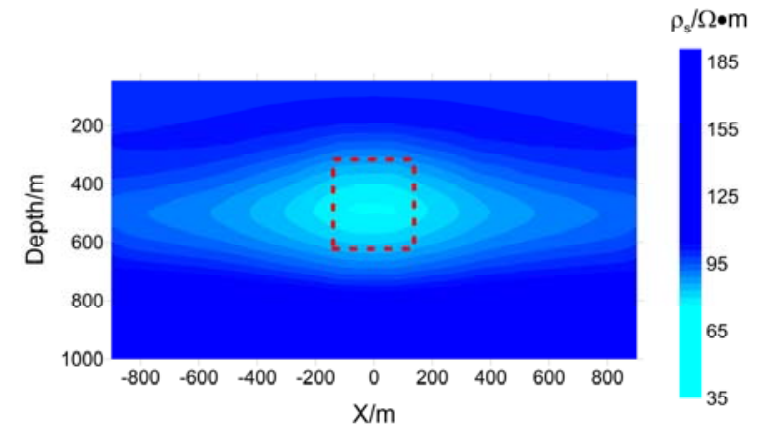

Fig. 4. apparent resistivity pseudo-section diagram

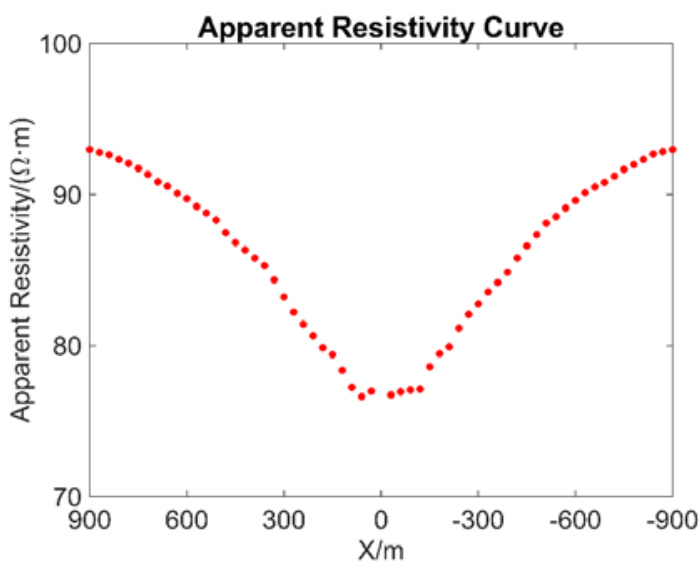

Fig. 5. the apparent resistivity curve of the profile $y=0 \mathrm{~m}$ 


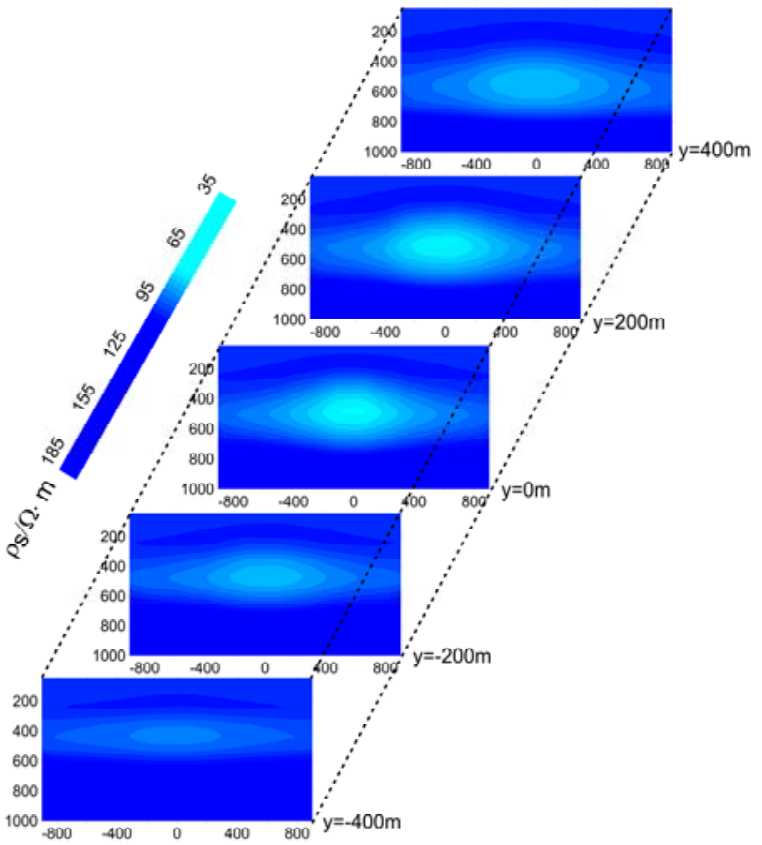

Fig. 6. Diagram of different slice faces

\section{CONCLUSION}

The finite element equation was derived from the vector wave equation, which was used to describe the secondary electric field equation. The unstructured mesh was used during the gridding process of the study area. The 3D code was validated by modeling the layered model and comparing its result with that of the IE method. The error margin between the results is under $3 \%$. This shows that the algorithm is accurate. Then, numerical modeling of a single conductive model was performed and the obtained electromagnetic response was analyzed. The obtained result was accurate. Hence, the derived algorithm can be used to perform numerical modeling of more complicated models.

\section{Acknowledgment}

This research was supported by R\&D of Key Instruments and Technologies for Deep Resources Prospecting (the National R\&D Projects for Key Scientific Instruments), Grant No. ZDYZ2012-1-05. Also, the author would like to thank professor Di Qingyun and Xue Guoqiang for their valuable suggestions.

\section{References}

[1] Hohmann G W. Three-dimensional induced polarization and electromagnetic modeling. Geophysics, 1975, 40(2): 309 324.

[2] Wannamaker P E, Stodt J A, Rijo L. Two-dimensional topographic response in magnetotullurics modeled using finite element. Geophysics, 1986, 51(11): 2131 2144.

[3] Mackie R L, Smith T J, Madden T R. 3D electromagnetic modeling using difference equations. Radio Sci. 1994, 29(5): 923 935.

[4] Di Qing-yun, Martyn Unsworth, Wang Miao-yue. 2.5D CSAMT modeling with the finite element method over 2D complex earth media. Chinese J. Geophys. (in Chinese), 2004, 47(4): 723 730.

[5] Wang Ruo, Wang Miao-yue, Lu Yuan-lin. Preliminary study on 3D3C CSAMT method modeling using finite element method. Progress in Geophysics, 2007, 22(2): 579 585.

[6] Xu Zhi-feng, Wu Xiao-ping. Constrolled source electromagnetic 3-D modeling in frequency domain by finite element method. Chinese J. Geophys. (in Chinese), 2010, 53(8): 1931 1939.

[7] Zhang Ji-feng, Tang Jin-tian, Yu Yan etc. Three dimensional controlled source electromagnetic numerical simulation based on electric field vector wave equation using finite element method. Chinese J. Geophys. (in Chinese), 2009, 52(12): 3132 3141.

[8] Ren Zheng-yong, Tang Jin-tian. Finite element modeling of 3D DC resistivity using locally refined unstructured meshes. Chinese J. Geophys. (in Chinese), 2009, 52(10): 2627 2634.

[9] Yuguo Li, Kerry Key. 2D marine controlled-source electromagnetic modeling: Part1- An adaptive finite-element algorithm. Geophysics, 2007, 72(2): WA51 WA62.

[10] Chen M S, Yan S. Analytical study on field zones, record rules, shadow and source overprint effects in CSAMT exploration. Chinese J. Geophys. (in Chinese), 2005, 48(4): 951 958.

[11] Kerry Key. 1D inversion of multicomponent, multifrequency marine CSEM data: Methodology and synthetic studies for resolving thin resistive layers. Geophysics, 2009, 74(2): F9 F20. 